\title{
Intramolecular dynamics of dsDNA confined to a quasi-one-dimensional nanochannel
}

\author{
Indresh Yadav, William Rosencrans, Rajib Basak, Jeroen A. van Kan ${ }^{\odot}$, and Johan R. C. van der Maarel ${ }^{*}{ }^{*}$ \\ Department of Physics, National University of Singapore, Singapore 117542, Singapore
}

(Received 8 November 2019; accepted 20 February 2020; published 11 March 2020)

\begin{abstract}
The intramolecular dynamics of double-stranded DNA confined to a long, straight, and rectangular channel with a cross-sectional diameter of $125 \mathrm{~nm}$ (two times the persistence length) is measured with fluorescence microscopy. The derived intermediate dynamic structure factors show predominant odd modes associated with end-to-end fluctuation and typical stretched exponential relaxation behavior pertaining to Rouse dynamics. Major confinement-induced slowing down of intramolecular fluctuation is observed, which may have important implications in biology and biotechnology. As predicted for free-draining in a quasi-one-dimensional conformation, the Rouse times show a cube rather than the classical, blob model predicted square dependence on the molecular weight.
\end{abstract}

DOI: 10.1103/PhysRevResearch.2.013294

\section{INTRODUCTION}

Stretching of double-stranded DNA (dsDNA) by confinement to a quasi-one-dimensional nanochannel with a cross-sectional diameter on the order of tens to hundreds of nanometers in conjunction with fluorescence imaging is an emerging technology with diverse (bio)technological applications [1-5]. It also serves as a platform for the investigation of the physical properties of single polymers [6-8]. Most of the work reported before concerns the molecular conformation, that is, how the equilibrated stretch responds to confinement and/or other environmental conditions. Investigations of the dynamical properties of confined polymers have focused on translocation, diffusivity, and segmental fluctuations [9-20]. Intramolecular fluctuations are thought to be important in DNA metabolism and gene expression regulation [21-23]. They also play a pivotal role in DNA motion control and sequence readout using nanopore or nanochannel devices $[24,25]$.

In early work on DNA confined to quasi-one-dimensional channels, Reisner et al. investigated the fluctuation in overall stretch [26]. The confined DNA was modeled as two beads joined by a spring with local hydrodynamic friction. This model is essentially a Rouse model but in one dimension and with neglect of higher order modes. A more detailed model including all modes was presented by Riehn and co-workers $[27,28]$. A key element is that intramolecular hydrodynamic interaction is screened beyond a distance scale on the order of the cross-sectional diameter of the channel. Indeed, for DNA confined to slitlike channels, Jones et al. showed that the screening length is proportional to channel height and

\footnotetext{
*johanmaarel@gmail.com

Published by the American Physical Society under the terms of the Creative Commons Attribution 4.0 International license. Further distribution of this work must maintain attribution to the author(s) and the published article's title, journal citation, and DOI.
}

that the autocorrelation matches a stretched exponential with stretching exponent $1 / 2$ pertaining to Rouse dynamics [15].

The task at hand is to map the Rouse model of a string of beads joined by springs to the real polymer chain confined to the quasi-one-dimensional channel. In the classical blob model proposed by Brochard and de Gennes, the beads are identified as blobs of channel diameter $D$ [29]. The longest relaxation (Rouse) time then becomes proportional to the square of the overall stretch $R$, that is, $\tau_{R} \propto D R^{2}$. Without any specific assumptions regarding the conformation of the molecule, the Rouse time can be expressed as

$$
\tau_{R}=\xi_{T} R^{2} /\left(\pi^{2} k_{B} T\right),
$$

with total chain friction $\xi_{T}$, mean square end-to-end distance $R^{2}$, and thermal energy $k_{B} T$ (see Appendix A) [30]. It is reasonable to assume that the total friction is proportional to the stretch, that is, $\xi_{T} \propto R$, because hydrodynamic interaction is screened beyond the diameter of the channel. In this conformation model-free approach, one obtains the markedly different result $\tau_{R} \propto R^{3}$. For a homogeneous density of segments along the channel, the stretch is extensive in the molecular weight $M$ [31]. The blob versus model-free implementation of the Rouse model can then be discerned by the $\tau_{R} \propto M^{2}$ vs $\tau_{R} \propto M^{3}$ dependence, respectively. Here, this will be done by measuring the Rouse time pertaining to bacteriophage $\lambda$-DNA and its dimeric and trimeric concatemers confined to a nanochannel through analysis of the fluctuation in segment density with fluorescence microscopy [32].

\section{METHODS}

\section{A. Chip fabrication}

Rectangular-shaped nanochannels with a length of $90 \mu \mathrm{m}$, a depth of $130 \pm 5 \mathrm{~nm}$, and a width of $120 \pm 5 \mathrm{~nm}$ (125-nm channel system) were fabricated by replication in polydimethylsiloxane with enhanced elasticity modulus (XPDMS) of a patterned master stamp [33-35]. The nanochannel part of the stamp was made in hydrogen silsesquioxane resist (Dow Corning, Midland, MI) using a lithography 
process with proton beam writing. An array of nanochannels is connected to two loading reservoirs through a superposing set of microchannels made in mr-DWL photoresist (Micro Resist Technology, Berlin, Germany) with a laser writer (Heidelberg micro PG 101). The heights and widths of the ridges in the master stamp were measured with atomic force microscopy (Dimension 3000, Veeco, Woodbury, NY) and scanning electron microscopy (JEOL JSM6700F), respectively. The master stamp was copied in the inorganic-organic hybrid polymer OrmoStamp (Micro Resist Technology) and coated with a 5-nm-thick teflon layer for perfect release of the replicated chips. The stamp was replicated in X-PDMS followed by curing at $333 \mathrm{~K}$ for $24 \mathrm{~h}$. Following plasma oxidation (Harrick, Ossining, NY), the X-PDMS replica was sealed with a glass coverslip.

\section{B. Sample preparation}

Bacteriophage $\lambda$-DNA (48.5 kbp, contour length of $16.5 \mu \mathrm{m})$ was purchased from New England Biolabs, Ipswich, MA. As received from the manufacturer the $\lambda$-phage DNA stock solution has a concentration of $0.5 \mathrm{~g}$ of DNA/1. The solvent was TE buffer, which is composed of $10 \mathrm{mM}$ Tris- $\mathrm{HCl}, \mathrm{pH} 8.0$, and $1 \mathrm{mM}$ EDTA. In order to remove naturally formed concatemers of $\lambda$-DNA, the stock solution was heated to $333 \mathrm{~K}$ for $10 \mathrm{~min}$, then rapidly cooled to $295 \mathrm{~K}$ by immersion in a water bath. Covalently bonded concatemers (dimers and trimers) of $\lambda$-DNA were prepared by joining the cohesive ends through phosphodiester bonds and enzymatic ligation with T4 DNA ligase (Promega, Madison, WI) [36]. The appropriate amounts of DNA and enzyme were mixed and ligation was carried out overnight at $277 \mathrm{~K}$. The reaction was inactivated by heating the sample to $338 \mathrm{~K}$ for $10 \mathrm{~min}$. Finally, the sample was dialyzed in a microdialyzer to remove excess salts and enzyme before redispersing in TE buffer. YOYO-1 fluorescence staining dye was purchased from Invitrogen, Carlsbad, CA. Prior to fluorescence imaging, DNA was stained with intercalating dye YOYO-1 at a ratio of one dye molecule for each four base pairs and kept overnight. One hour before the measurement, the oxygen scavenger $\beta$ mercaptoethanol (Sigma-Aldrich, St. Louis, MO) was added $(4 \% \mathrm{v} / \mathrm{v})$ to prevent photobleaching.

\section{Fluorescence imaging}

The solution of the stained DNA molecules was pipetted into the two loading reservoirs connected by the array of nanochannels. The DNA molecules were subsequently driven into the channels by electrophoresis. For this purpose, two platinum electrodes were immersed in the reservoirs and connected to a power supply with a voltage in the range $0.1-$ $10 \mathrm{~V}$ (Keithley, Cleveland, $\mathrm{OH}$ ). Once the DNA molecules were brought inside the nanochannels, the electric field was switched off and the molecules were allowed to relax to their equilibrium state for 2-5 min. The stained DNA molecules were visualized with a Nikon Eclipse Ti inverted fluorescence microscope equipped with a $200-\mathrm{mW} / 488-\mathrm{nm}$ laser and a $100 \times$ oil immersion objective (numerical aperture 1.49). Movie clips capturing the fluctuations of individual molecules were recorded with an electron-multiplying charged coupled device camera (Andor iXon X3). The image pixel size of $0.16 \times 0.16 \mu \mathrm{m}^{2}$ was calibrated with the help of a metric ruler. Single molecules of the appropriate stretch (mono, di-, or trimers) were selected. The video clips were analyzed using home-developed scripts in MATLAB, R2019b (MathWorks, Natick, MA).

\section{RESULTS AND DISCUSSION}

Nanofluidic devices featuring rectangular-shaped channels with a length of $90 \mu \mathrm{m}$, a depth of $130 \pm 5 \mathrm{~nm}$, and a width of $120 \pm 5 \mathrm{~nm}$ were made of polydimethylsiloxane with enhanced elasticity modulus (X-PDMS) as described in the Methods section [33-35]. The average cross-sectional diameter of $125 \mathrm{~nm}$ is about two times the stained DNA persistence length of $60 \mathrm{~nm}$ [37]. Covalently bonded concatemers $(\lambda$-DNA 2 dimers and $\lambda$-DNA 3 trimers) of $\lambda$-DNA were prepared by enzymatic ligation [36]. The molecules were dispersed in TE buffer to a concentration of $0.3 \mathrm{mg}$ of DNA/1 and brought into the array of nanochannels by electrophoresis. After switching off the electric field, the molecules relax to their equilibrium state within $120 \mathrm{~s}$. Video recording was started 2-5 min after the molecules were brought into the channels and the clips lasted for another 3-7 min. The frame rate was 50 or $20 \mathrm{fps}$, with the lower frame rate and longer clip duration for $\lambda$-DNA 3 . Overexposure was avoided by careful adjustment of camera gain and exposure time. DNAs with a stretch being the mean value minus two times the standard deviation were ignored. We selected and further analyzed a pool of 80,60 , and $60 \lambda$-DNA, $\lambda$-DNA 2 , and $\lambda$-DNA 3 molecules, respectively. A fresh chip was used for every 20 molecules. No sticking of DNA to the X-PDMS surface was observed. Typical fluorescence images of single $\lambda$-DNA,

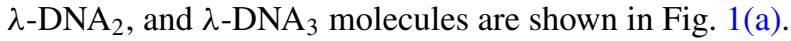

The video clips were analyzed by obtaining the $z$-axis projected intensity profile $\rho(z, t)$ along the channel for each frame. Effects of photobleaching were corrected by normalization of each frame's intensity profile to its total intensity. The time-averaged profile $\bar{\rho}(z)$ was obtained by averaging over the duration of the clip. A profile of the fluctuation in intensity was then obtained by subtraction of the timeaveraged intensity profile from the temporal profile, that is, $\delta \rho(z, t)=\rho(z, t)-\bar{\rho}(z)$. An example of a thus obtained kymograph pertaining to $\lambda$-DNA is shown in Fig. $1(\mathrm{~b})$. With the assumption that the intensity is proportional to DNA density, such a kymograph represents density fluctuation $\delta \rho(z, t)$. Notice that the molecules are entropically trapped and that there is no appreciable net drift of the center of mass.

Segment density correlations are analyzed through evaluation of the Van Hove correlation function

$$
G(z, \tau)=\left\langle\delta \rho\left(z^{\prime}, t\right) \delta \rho\left(z^{\prime}+z, t+\tau\right)\right\rangle / N,
$$

where the chevrons denote an average over $z^{\prime}$ and $t$ [38]. $G(z, \tau)$ is normalized to the total number of segments $N=$ $\int_{-\infty}^{\infty} d z \bar{\rho}(z)$. An example based on the evaluation of the kymographs pertaining to $\lambda$-DNA is shown in Fig. 1(c) (average of 80 molecules). Correlations in space and time are more conveniently discussed in terms of the intermediate dynamic structure factor $F(k, \tau)$. The dynamic structure factor can be obtained through one-dimensional Fourier transformation of 
(a)

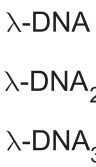

(b)

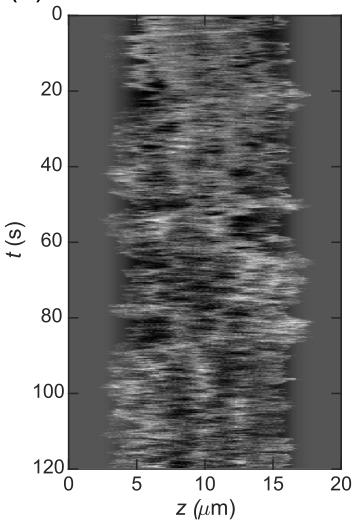

(c)

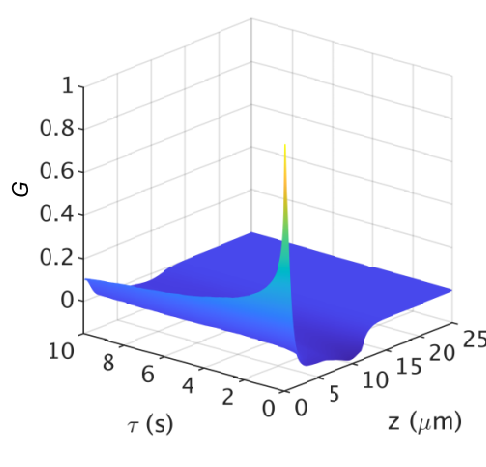

FIG. 1. (a) Fluorescence images of $\lambda$-DNA, dimeric $\lambda$-DNA 2 concatemer, and trimeric $\lambda$-DNA 3 concatemer. (b) Kymograph of density fluctuation $\delta \rho(z, t)$ for $\lambda$-DNA confined to a $125-\mathrm{nm}$ channel. The total duration of the clip is $2 \mathrm{~min}$. (c) The corresponding Van Hove correlation function $G(z, \tau)$ (average of 80 molecules).
$G(z, \tau)$ along the channel $z$ coordinate according to

$$
F(k, \tau)=\int_{-\infty}^{\infty} d z \exp (-i k z) G(z, \tau)
$$

(see Appendix B). The results pertaining to an average of

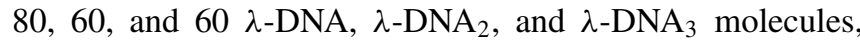
respectively, are displayed in Fig. 2. Notice the decrease in time decay with increasing molecular weight. The singularity at $k=0$ is suppressed, because the time-averaged profile has been subtracted from the temporal profiles. Furthermore, the $k$ axis has been scaled by multiplication with stretch $R$ as obtained from the half wavelength of the fundamental (see below).

Intramolecular hydrodynamic interaction is screened beyond a distance scale of around the channel diameter $(125 \mathrm{~nm})$ [15]. Accordingly, the confined polymer can be considered a sequence of freely drained beads joined by springs packed in a linear array [27,28]. This model is essentially the Rouse model for polymer dynamics but in one dimension. The pertinent equations are summarized in Appendix A. Irrespective of molecular weight, the predominant features of $F(k, \tau)$ are the odd Rouse modes associated with end-to-end fluctuation [30]. The positions of these modes are at $k_{p}=p /(2 R)$ with mode number $p$ and half wavelength of the fundamental $R$. Measured values of $k_{p}$ vs $p$ are shown in Fig. 3(a). Linear least-squares fits intersecting the origin confirm that the predominant features in $F(k, \tau)$ are indeed odd modes. The values of $R$ and their standard deviations resulting from (a)
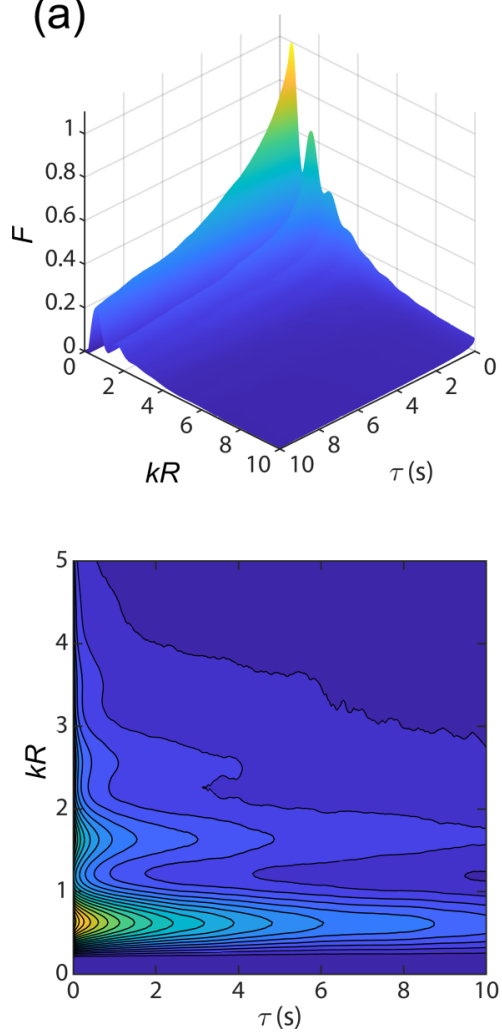

(b)
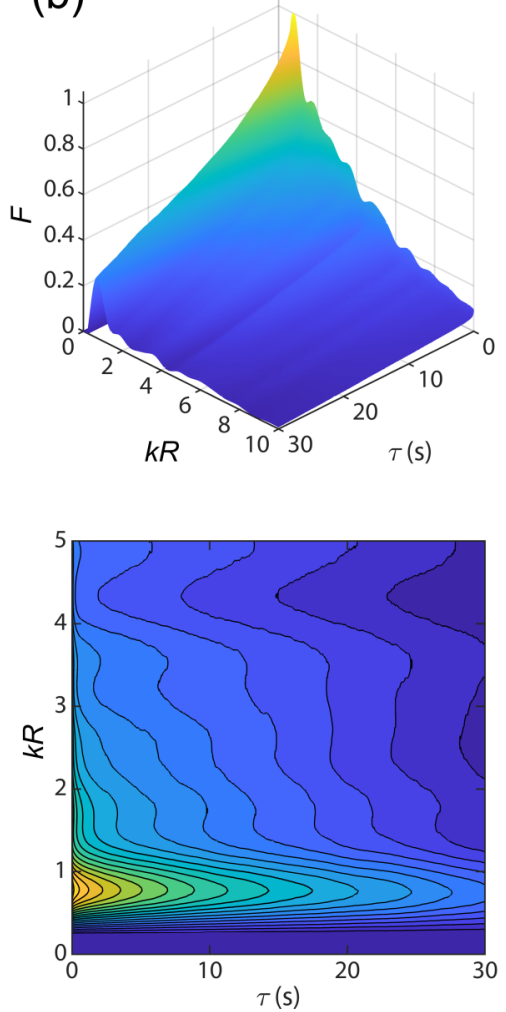

(c)
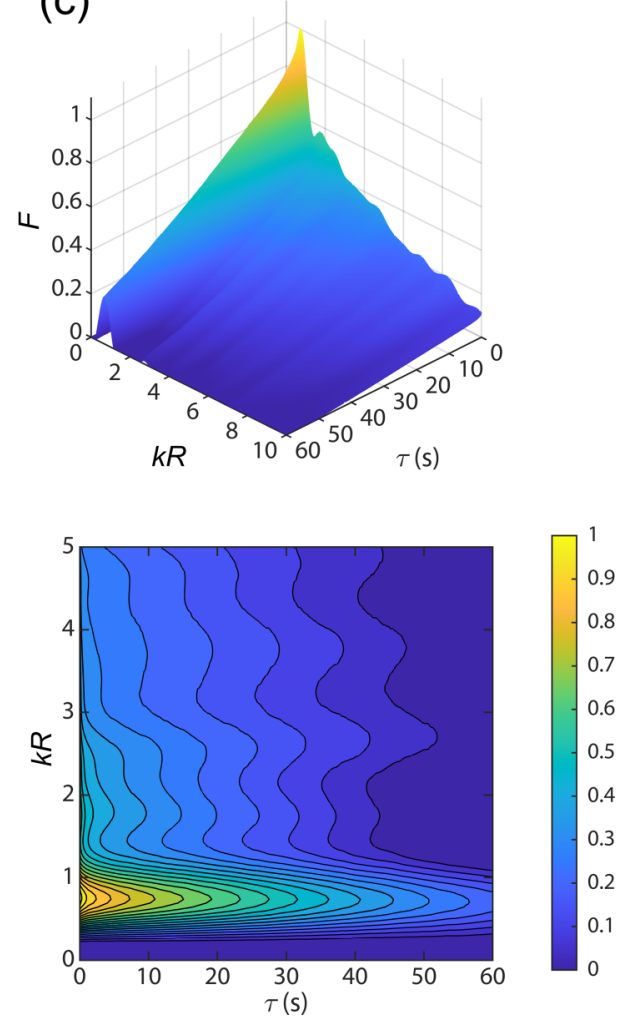

FIG. 2. (a) Surface (top) and contour (bottom) plots of the intermediate dynamic structure factor $F(k, \tau)$ of $\lambda$-DNA confined to a 125 -nm channel. (b) As in panel (a), but for dimeric $\lambda$-DNA 2 concatemer. (c) As in panel (a), but for trimeric $\lambda$-DNA 3 concatemer. Notice that the $k$ axis has been scaled by multiplication with stretch $R$. 


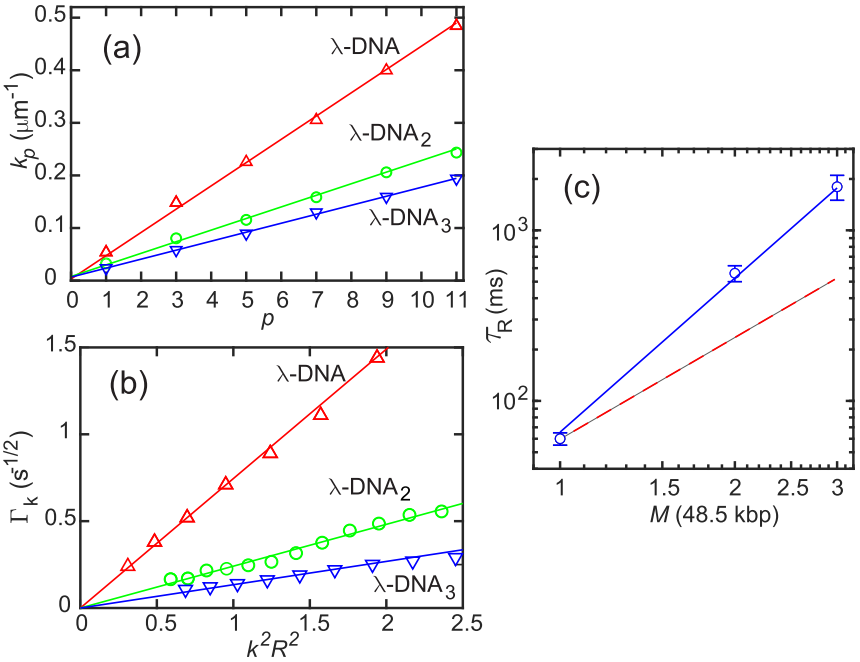

FIG. 3. (a) Positions of the maxima $k_{p}$ vs mode number $p$ for $\lambda$-DNA $\left(\Delta\right.$, red), $\lambda$-DNA $2(\bigcirc$, green $)$, and $\lambda-\mathrm{DNA}_{3}(\nabla$, blue). The solid lines denote linear fits giving the half wavelength of the fundamental $R$. (b) As in panel (a), but for the decay constants $\Gamma_{k}$ vs $k^{2} R^{2}$ resulting from a fit of a stretched exponential to the initial decay of $F(k, \tau)$. The solid lines represent a linear fit with optimized Rouse times. (c) Rouse time $\tau_{R}$ vs molecular weight $M$ in units of $48.5 \mathrm{kbp}$. The solid blue and dashed red lines represent $\tau_{R} \propto M^{3}$ and $\tau_{R} \propto M^{2}$ variation, respectively.

the regression analysis are collected in Table I. $R$ values for the concatemers are in reasonable agreement with the relevant multiples of the value of $R$ for the $\lambda$-DNA monomer. Notice that the stretch is about half the contour length, which implies that the molecules are still coiled but close to the transition from the blob to the deflection regime as indicated by Monte Carlo simulation [8].

$F(k, \tau)$ shows a relatively slow relaxation on a timescale from seconds to tens of seconds with the longer timescale for the larger molecular weight (see Fig. 2). The lag time dependence of $F(k, \tau)$ depends on the particulars of the dynamics (see Appendix C) [30]. For long times, $F(k, \tau)$ is determined by diffusion of the center of mass, that is, $F(k, 0) \exp \left(-k^{2} D_{\mathrm{cm}} \tau\right)$ with diffusion constant $D_{\mathrm{cm}}$. In the case of Rouse dynamics at shorter times, $F(k, \tau)$ takes the form of a stretched exponential, that is,

$$
F(k, \tau)=F(k, 0) \exp \left(-\Gamma_{k} \tau^{1 / 2}\right),
$$

with decay constant

$$
\Gamma_{k}=k^{2} R^{2} /\left(\pi^{3 / 2} \tau_{R}^{1 / 2}\right),
$$

TABLE I. Molecular weight $M$, dye-corrected contour length $L$, half wavelength of the fundamental $R$, and Rouse time $\tau_{R}$ for $\lambda$-DNA and its di- and trimeric concatemers confined to a $125-\mathrm{nm}$ channel.

\begin{tabular}{lcccc}
\hline \hline & $\begin{array}{c}M \\
(\mathrm{kbp})\end{array}$ & $\begin{array}{c}L \\
(\mu \mathrm{m})\end{array}$ & $\begin{array}{c}R \\
(\mu \mathrm{m})\end{array}$ & $\begin{array}{c}\tau_{R} \\
(\mathrm{~ms})\end{array}$ \\
\hline$\lambda$-DNA & 48.5 & 21.4 & $11.3 \pm 0.1$ & $60 \pm 5$ \\
$\lambda$-DNA & 97 & 42.8 & $22.7 \pm 0.2$ & $560 \pm 60$ \\
$\lambda$-DNA & 145.5 & 64.2 & $29.9 \pm 0.6$ & $1800 \pm 300$ \\
\hline \hline
\end{tabular}
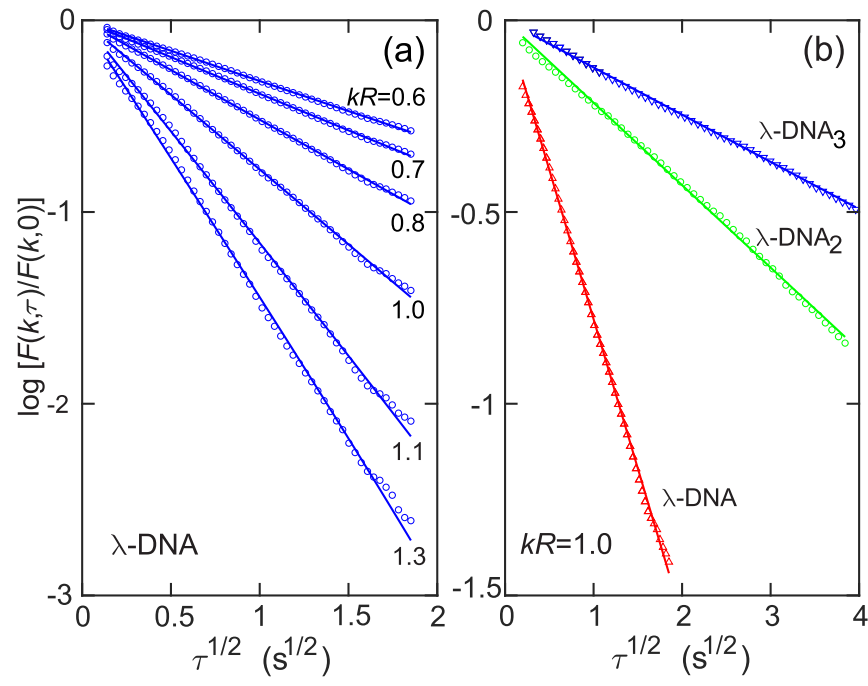

FIG. 4. (a) $\log [F(k, \tau) / F(k, 0)]$ vs $\tau^{1 / 2}$ for $\lambda$-DNA and $k R=$ $0.6,0.7,0.8,1.0,1.1$, and 1.3 from top to bottom. The solid lines denote a stretched exponential fit. (b) As in panel (a), but for $\lambda-\mathrm{DNA}_{3}$ ( $\nabla$, blue), $\lambda$-DNA 2 ( $\bigcirc$, green), and $\lambda$-DNA $(\triangle$, red), and $k R=1.0$ from top to bottom

and Rouse time $\tau_{R}[15,30,39]$. A stretched exponential was fitted to the initial decay for $k R$ values in the range $0.6<$ $k R<1.6$ with nonlinear least-squares regression. For larger values of $k R$, the fits become unreliable due to poorer statistics. $F(k, \tau)$ shows a very fast decay over a few lag times due to camera noise. The corresponding data points were excluded from the fits. Results of the fits pertaining to $\lambda$-DNA for a range of values of $k R$ are shown in Fig. 4(a). The results for $\lambda$-DNA and its di- and trimeric concatemers but for a single value of $k R=1.0$ are displayed in Fig. 4(b). Perfect stretched exponential behavior with a stretched exponent of $1 / 2$ is observed. The fitted values of $\Gamma_{k}$ vs $k^{2} R^{2}$ are set out in Fig. 3(b). Irrespective of molecular weight, an origin intersecting, linear dependence is observed. The Rouse times and their standard deviations resulting from least-squares regression according to Eq. (5) are also collected in Table I.

For $\lambda$-DNA confined to the $125-\mathrm{nm}$ channel, the value of $\tau_{R}$ ( $\left.\sim 60 \mathrm{~ms}\right)$ is comparable to those obtained for reptating DNA in the semidilute regime (30-100 ms) [40]. Following a threefold increase in molecular weight and, hence, stretch, intramolecular dynamics is slowed down considerably with a two orders of magnitude increase in value of $\tau_{R}(\sim 2 \mathrm{~s})$. As predicted for Rouse dynamics in a quasi-one-dimensional, straight channel, the Rouse time shows $\tau_{R} \propto M^{3}$ variation [see Fig. 3(c)]. This confirms a total chain friction proportional to the stretch (screening of intramolecular hydrodynamic interaction) and a stretch proportional to the molecular weight (homogeneous segment distribution).

\section{SUMMARY}

The dynamics of DNA confined to a channel with a diameter of about two times the persistence length was measured with fluorescence microscopy. From the fluctuation in intensity, we derived the intermediate dynamic structure factor. Irrespective of DNA molecular weight, the structure 
factor shows predominant odd order modes associated with end-to-end fluctuation. The stretched exponential decay for shorter times with stretching exponent $1 / 2$ is a hallmark of Rouse dynamics. Contrary to the prediction based on the blob model, the experimentally derived Rouse times agree with a cube dependence on the molecular weight. Note that the $\tau_{R} \propto$ $M^{3}$ dependence is unique for free draining in a quasi-onedimensional conformation. For a self-avoiding sequence in two dimensions $\left(R \propto M^{3 / 4}\right)$, the model-free implementation implies $\tau_{R} \propto M^{5 / 2}$. In the case of Rouse dynamics in the threedimensional semidilute regime $\left(R \propto M^{1 / 2}\right)$, one recovers the classical result $\tau_{R} \propto M^{2}$. It should also be noted that the $\tau_{R} \propto$ $M^{3}$ dependence has been derived without any specific assumptions regarding the conformation of the molecule. However, the scaling law will no longer hold for any inhomogeneous distribution of segments along the channel with a positiondependent friction and/or elasticity constant. We surmise that the major slowing down of intramolecular thermal fluctuations by confinement to an elongated nanospace has important implications in DNA metabolism and gene expression regulation as well as motion control in DNA sequence readout devices.

\section{ACKNOWLEDGMENT}

This research was supported by Singapore Ministry of Education Academic Research Fund Tier 1.

\section{APPENDIX A: ONE-DIMENSIONAL ROUSE MODEL}

The chain confined to the channel is modeled as a sequence of $N$ beads connected by springs. We follow the formalism of Doi and Edwards, but modified for fluctuation in one dimension [30]. In the Rouse model, hydrodynamic interaction among the beads is neglected. The Langevin equation becomes a linear equation for bead position $z_{n}$, that is,

$$
\xi \frac{d z_{n}}{d t}=-k\left(2 z_{n}-z_{n+1}-z_{n-1}\right)+f_{n},
$$

with spring constant $k=k_{B} T / b^{2}$, friction per bead $\xi$, and random force $f_{n}$. Bead position $z_{n}$ can be expressed in terms of the Rouse modes $X_{p}$ according to

$$
z_{n}=X_{0}+2 \sum_{p=1}^{\infty} X_{p} \cos \left(\frac{p \pi n}{N}\right),
$$

where $X_{0}$ represents the position of the center of mass

$$
X_{0}=\frac{1}{N} \int_{0}^{N} d n z_{n} .
$$

The correlation of the Rouse modes (in one dimension) is given by

$$
\left\langle X_{p}(\tau) X_{q}(0)\right\rangle=\delta_{p q} \frac{N b^{2}}{2 \pi^{2} p^{2}} \exp \left(-p^{2} \tau / \tau_{R}\right),
$$

with Rouse relaxation time $\tau_{R}$. The Rouse time reads

$$
\tau_{R}=\frac{\xi N^{2} b^{2}}{\pi^{2} k_{B} T} \text {. }
$$

According to Eq. (A2), the temporal end-to-end stretch $R(t)=z_{N}(t)-z_{0}(t)$ can be expressed in terms of a sum of odd Rouse modes

$$
R(t)=-4 \sum_{k=0}^{\infty} X_{2 k+1}(t),
$$

with time correlation

$$
\begin{array}{r}
\langle R(\tau) R(0)\rangle=16 \sum_{k=0}^{\infty}\left\langle X_{2 k+1}(0) X_{2 k+1}(\tau)\right\rangle \\
=\frac{8 N b^{2}}{\pi^{2}} \sum_{k=0}^{\infty} \frac{1}{(2 k+1)^{2}} \exp \left[-(2 k+1)^{2} \tau / \tau_{R}\right] .
\end{array}
$$

For $\tau=0$, we obtain the mean square end-to-end distance $R^{2}=N b^{2}$. In terms of model-free parameters, the Rouse time takes the form

$$
\tau_{R}=\frac{\xi_{T} R^{2}}{\pi^{2} k_{B} T},
$$

with total friction of the chain $\xi_{T}=N \xi$.

\section{APPENDIX B: SPACE AND TIME CORRELATION}

The DNA molecule is confined to a quasi-one-dimensional channel with a $z$-axis projected and time-dependent density of the segments

$$
\rho(z, t)=\sum_{m=1}^{N} \delta\left[z-z_{m}(t)\right] .
$$

Here, $z_{m}(t)$ is the $z$ coordinate along the channel of the $m$ th segment at time $t$ and the summation runs over the total number of segments $N$. The latter follows from integration of the density along the channel

$$
N=\int_{-\infty}^{\infty} d z \rho(z) .
$$

In order to remove the singularity at $k=0$, we will consider the density fluctuation, that is, $\delta \rho(z, t)=\rho(z, t)-\bar{\rho}(z)$ with $\bar{\rho}(z)$ the time-averaged density profile.

The intermediate dynamic structure factor reads

$$
F(k, \tau)=\frac{1}{N}\left\langle\rho_{k}(t) \rho_{-k}(t+\tau)\right\rangle,
$$

with spatial Fourier transform of the segment density fluctuation

$$
\rho_{k}(t)=\int_{-\infty}^{\infty} d z \exp (i k z) \delta \rho(z, t) .
$$

The intermediate dynamic structure factor can be expressed in terms of segment density correlation in space and time according to

$$
\begin{aligned}
F(k, \tau)= & \frac{1}{N} \int_{-\infty}^{\infty} d z^{\prime \prime} \int_{-\infty}^{\infty} d z^{\prime} \exp \left[-i k\left(z^{\prime}-z^{\prime \prime}\right)\right] \\
& \times\left\langle\delta \rho\left(z^{\prime \prime}, t\right) \delta \rho\left(z^{\prime}, t+\tau\right)\right\rangle .
\end{aligned}
$$

With $z=z^{\prime}-z^{\prime \prime}$

$$
F(k, \tau)=\int_{-\infty}^{\infty} d z \exp (-i k z) G(z, \tau),
$$


where $G(z, \tau)$ denotes the Van Hove correlation function

$$
G(z, \tau)=\frac{1}{N}\left\langle\delta \rho\left(z^{\prime}, t\right) \delta \rho\left(z^{\prime}+z, t+\tau\right)\right\rangle
$$

and the chevrons denote an average over $z^{\prime}$ and $t$.

\section{APPENDIX C: INTERMEDIATE DYNAMIC STRUCTURE FACTOR}

For Gaussian fluctuation, the intermediate dynamic structure factor takes the form [30]

$$
F(k, \tau)=\frac{1}{N} \sum_{m, n=1}^{N} \exp \left(-k^{2} \phi_{m n} / 2\right),
$$

with $\phi_{m n}=\left\langle\left[z_{m}(\tau)-z_{n}(0)\right]^{2}\right\rangle$. In the limit of long times, $\tau \gg$ $\tau_{R}$, the intermediate dynamic structure factor is determined by diffusion of the center of mass, that is,

$$
\phi_{m n}=\left\langle\left[X_{0}(\tau)-X_{0}(0)\right]^{2}\right\rangle=2 D_{\mathrm{cm}} \tau,
$$

so that,

$$
\begin{aligned}
F(k, \tau) & =N \exp \left(-k^{2} D_{\mathrm{cm}} \tau\right) \\
& =F(k, 0) \exp \left(-k^{2} D_{\mathrm{cm}} \tau\right) .
\end{aligned}
$$

For shorter times, Rouse dynamics is observed and the time dependence of the dynamic structure factor takes the form of a stretched exponential $[30,39]$

$$
F(k, \tau)=F(k, 0) \exp \left(-\Gamma_{k} \tau^{1 / 2}\right),
$$

with decay constant

$$
\Gamma_{k}=\frac{k^{2} R^{2}}{\pi^{3 / 2} \tau_{R}^{1 / 2}}
$$

[1] K. Jo, D. M. Dhingra, T. Odijk, J. J. de Pablo, M. D. Graham, R. Runnheim, D. Forrest, and D. C. Schwartz, A single-molecule barcoding system using nanoslits for DNA analysis, Proc. Natl. Acad. Sci. USA 104, 2673 (2007).

[2] E. Lam, A. Hastie, C. Lin, D. Ehrlich, S. Das, M. Austin, P. Deshpande, H. Cao, N. Nagarajan, M. Xiao, and P. Kwok, Genome mapping on nanochannel arrays for structural variation analysis and sequence assembly, Nat. Biotechnol. 30, 771 (2012).

[3] K. Frykholm, L. K. Nyberg, and F. Westerlund, Exploring DNA-protein interactions on the single DNA molecule level using nanofluidic tools, Integr. Biol. 9, 650 (2017).

[4] V. Müller and F. Westerlund, Optical DNA mapping in nanofluidic devices: Principles and applications, Lab Chip 17, 579 (2017).

[5] D. Ödman, E. Werner, K. D. Dorfman, C. R. Doering, and B. Mehlig, Distribution of label spacings for genome mapping in nanochannels, Biomicrofluidics 12, 034115 (2018).

[6] W. Reisner, J. N. Pedersen, and R. H. Austin, DNA confinement in nanochannels: Physics and biological applications, Rep. Prog. Phys. 75, 106601 (2012).

[7] J. R. C. van der Maarel, C. Zhang, and J. A. van Kan, A nanochannel platform for single DNA studies: From crowding, protein DNA interaction, to sequencing of genomic information, Isr. J. Chem. 54, 1573 (2014).

[8] L. Dai, C. B. Renner, and P. S. Doyle, The polymer physics of single DNA confined in nanochannels, Adv. Colloid Interface Sci. 232, 80 (2016).

[9] C. T. A. Wong and M. Muthukumar, Polymer translocation through a cylindrical channel, J. Chem. Phys. 128, 154903 (2008).

[10] P. Fanzio, C. Manneschi, E. Angeli, V. Mussi, G. Firpo, L. Ceseracciu, L. Repetto, and U. Valbusa, Modulating DNA translocation by a controlled deformation of a PDMS nanochannel device, Sci. Rep. 2, 791 (2012).

[11] J. Zhou, Y. Wang, L. D. Menard, S. Panyukov, M. Rubinstein, and J. M. Ramsey, Enhanced nanochannel translocation and localization of genomic DNA molecules using three-dimensional nanofunnels, Nat. Commun. 8, 807 (2017).
[12] L. D. Menard and J. M. Ramsey, Electrokinetically-driven transport of DNA through focused ion beam milled nanofluidic channels, Anal. Chem. 85, 1146 (2013).

[13] D. J. Bonthuis, C. Meyer, D. Stein, and C. Dekker, Conformation and Dynamics of DNA Confined in Slitlike Nanofluidic Channels, Phys. Rev. Lett. 101, 108303 (2008).

[14] A. Balducci, P. Mao, J. Han, and P. Doyle, Double-stranded DNA diffusion in slitlike nanochannels, Macromolecules 39 , 6273 (2006).

[15] J. J. Jones, J. R. C. van der Maarel, and P. S. Doyle, Intrachain Dynamics of Large dsDNA Confined to Slitlike Channels, Phys. Rev. Lett. 110, 068101 (2013).

[16] J. O. Tegenfeldt, C. Prinz, H. Cao, S. Chou, W. W. Reisner, R. Riehn, Y. M. Wang, E. C. Cox, J. C. Sturm, P. Silberzan, and R. H. Austin, The dynamics of genomic-length DNA molecules in 100-nm channels, Proc. Natl. Acad. Sci. USA 101, 10979 (2004).

[17] S. L. Levy, J. T. Mannion, J. Cheng, C. H. Reccius, and H. G. Craighead, Entropic unfolding of DNA molecules in nanofluidic channels, Nano Lett. 8, 3839 (2008)

[18] C. H. Reccius, S. M. Stavis, J. T. Mannion, L. P. Walker, and H. G. Craighead, Conformation, length, and speed measurements of electrodynamically stretched DNA in nanochannels, Biophys. J. 95, 273 (2008).

[19] A. Jain, J. Sheats, J. G. Reifenberger, H. Cao, and K. D. Dorfman, Modeling the relaxation of internal DNA segments during genome mapping in nanochannels, Biomicrofluidics 10, 054117 (2016).

[20] D. Gupta, A. B. Bhandari, and K. D. Dorfman, Evaluation of blob theory for the diffusion of DNA in nanochannels, Macromolecules 51, 1748 (2018).

[21] Y. M. Wang, R. H. Austin, and E. C. Cox, Single Molecule Measurements of Repressor Protein 1D Diffusion on DNA, Phys. Rev. Lett. 97, 048302 (2006).

[22] A. Mondal and A. Bhattacherjee, Searching target sites on DNA by proteins: Role of DNA dynamics under confinement, Nucleic Acids Res. 43, 9176 (2015). 
[23] E. Chow and J. Skolnick, DNA internal motion likely accelerates protein target search in a packed nucleoid, Biophys. J. 112, 2261 (2017).

[24] S. Carson and M. Wanunu, Challenges in DNA motion control and sequence readout using nanopore devices, Nanotechnology 26, 074004 (2015).

[25] J. Sheats, J. G. Reifenberger, H. Cao, and K. D. Dorfman, Measurements of DNA barcode label separations in nanochannels from time-series data, Biomicrofluidics 9, 064119 (2015).

[26] W. Reisner, K. J. Morton, R. Riehn, Y. M. Wang, Z. Yu, M. Rosen, J. C. Sturm, S. Y. Chou, E. Frey, and R. H. Austin, Statics and Dynamics of Single DNA Molecules Confined in Nanochannels, Phys. Rev. Lett. 94, 196101 (2005).

[27] J. H. Carpenter, A. Karpusenko, J. Pan, S. F. Lim, and R. Riehn, Density fluctuations dispersion relationship for a polymer confined to a nanotube, Appl. Phys. Lett. 98, 253704 (2011).

[28] A. Karpusenko, J. H. Carpenter, C. Zhou, S. F. Lim, J. Pan, and R. Riehn, Fluctuation modes of nanoconfined DNA, J. Appl. Phys. 111, 024701 (2012).

[29] F. Brochard and P. G. de Gennes, Dynamics of confined polymer chains, J. Chem. Phys. 67, 52 (1977).

[30] M. Doi and S. F. Edwards, The Theory of Polymer Dynamics (Oxford University Press, New York, 1988), Vol. 73.

[31] M. Daoud and P. G. de Gennes, Statistics of macromolecular solutions trapped in small pores, J. Phys. (Paris) 38, 85 (1977).
[32] A. E. Cohen and W. E. Moerner, Principal-components analysis of shape fluctuations of single DNA molecules, Proc. Natl. Acad. Sci. USA 104, 12622 (2007).

[33] J. A. van Kan, A. A. Bettiol, and F. Watt, Proton beam writing of three-dimensional nanostructures in hydrogen silsesquioxane, Nano Lett. 6, 579 (2006).

[34] C. Zhang, F. Zhang, J. A. van Kan, and J. R. C. van der Maarel, Effects of electrostatic screening on the conformation of single DNA molecules confined in a nanochannel, J. Chem. Phys. 128, 225109 (2008).

[35] J. A. van Kan, C. Zhang, P. P. Malar, and J. R. C. van der Maarel, High throughput fabrication of disposable nanofluidic lab-onchip devices for single molecule studies, Biomicrofluidics 6 , 036502 (2012).

[36] R. J. Bauer, A. Zhelkovsky, K. Bilotti, L. E. Crowell, T. C. Evans, Jr., L. A. McReynolds, and G. J. Lohman, Comparative analysis of the end-joining activity of several DNA ligases, PLoS One 12, e0190062 (2017).

[37] B. Kundukad, J. Yan, and P. Doyle, Effect of YOYO-1 on the mechanical properties of DNA, Soft Matter 10, 9721 (2014).

[38] J.-P. Hansen and I. R. McDonald, Theory of Simple Liquids (Elsevier, New York, 1990).

[39] P. G. de Gennes, Quasi-elastic scattering of neutrons by dilute polymer solutions: I. Free-draining limit, Phys. Phys. Fiz. 3, 37 (1967).

[40] Z. Gong and J. R. C. van der Maarel, Translational and reorientational dynamics of entangled DNA, Macromolecules 47, 7230 (2014). 